PROCEEDINGS OF THE

AMERICAN MATHEMATICAL SOCIETY

Volume 34, Number 2, August 1972

\title{
ERRATA FOR TWO PAPERS OF STITZINGER
}

\author{
ERNEST L. STITZINGER
}

There is a mistake in each of [3] and [4]. The purpose of this note is to correct the error in [3] and to salvage what can be saved in [4]. In each case the notation will be that of the paper under discussion.

Professor Homer Bechtell has kindly informed me of an error in the proof of the Theorem of [3] which occurs in the case $M G^{\mathbf{1}} \subset G$. The theorem is true however by altering the proof at this point.

Assume that $M \subset M G^{1}=M_{1} \subset G . M_{1}$ is an invariant subgroup of $G$, and since $G^{1} \subset \operatorname{Soc}(G), G^{1}$ is a direct sum of minimal invariant subgroups of $G$. By Clifford's theorem (p. 70 of [1]), each minimal invariant subgroup of $G$ is either $M_{1}$-central or $M_{1}$-hypereccentric, and hence

$$
G^{1}=\left(G^{1} \cap Z\left(M_{1}\right)\right) \times\left[G^{1}, M_{1}\right] .
$$

Let $C$ be a Carter subgroup of $G$ and let $C_{1}=\left(C \cap M_{1}\right) \times\left(G^{1} \cap Z\left(M_{1}\right)\right)$. By the corollary to Lemma $2, C_{1}$ is a Carter subgroup of $M_{1}$, since $\operatorname{Fr}\left(M_{1}\right)=1$. But $C$ and $G^{1}$ are elementary abelian, hence $C_{1}$ is also. By the minimality of $G, M_{1}$ is elementary, hence $\operatorname{Fr}(M)=1$.

In [4], the claim in Lemma 3 that $M$ is a subgroup, is not true in general. If $A$ is elementary abelian, then $M$ is a subgroup, hence the theorem holds in that case. The theorem also holds for the following conditions on $\mathfrak{X}$. Let $\mathfrak{X}$ be the class of nilpotent groups $N$ with the following properties:

(1) $Z_{1}(N)$ is a cyclic $p$-group.

(2) There exists an elementary abelian characteristic subgroup $A$ of $N$ such that $A \nsubseteq Z_{1}(N)$ and $A \subseteq Z_{2}(N)$.

Here we let $K=A \cap Z_{1}(N)$ and evidently $|K|=p$. Now the proofs in the paper may be used with $K$ replacing $Z_{1}(N)$. Note that the existence of $A$ is equivalent to the existence of a noncyclic characteristic elementary abelian subgroup. Now the $p$-groups which do not have such a subgroup are given in Satz 13.10, Kapitel III of [2].

\section{REFERENCES}

1. D. Gorenstein, Finite groups, Harper and Row, New York, 1968. MR 38 \#229.

2. B. Huppert, Endliche Gruppen. I, Die Grundlehren der math. Wissenschaften, Band 134, Springer-Verlag, Berlin and New York, 1967. MR 37 \#302.

3. E. Stitzinger, On elementary groups, Proc. Amer. Math. Soc. 26 (1970), 236-238. MR $42 \# 376$.

4. —, A nonembedding theorem for finite groups, Proc. Amer. Math. Soc. 25 (1970), 124-126. MR 41 \#3581.

Received by the editors January 4, 1971 and, in revised form, January 24, 1972. 